\title{
Continuous Global Evidence-Based Bayesian Modality Fusion for Simultaneous Tracking of Multiple Objects
}

\author{
Jamie Sherrah \\ Shaogang Gong \\ Department of Computer Science, Queen Mary, University of London \\ LONDON E1 4NS, UK, jamie|sgg@dcs.qmw.ac.uk
}

\begin{abstract}
Robust, real-time tracking of objects from visual data requires probabilistic fusion of multiple visual cues. Previous approaches have either been ad hoc or relied on a Bayesian network with discrete spatial variables which suffers from discretisation and computational complexity problems. We present a new Bayesian modality fusion network that uses continuous domain variables. The network architecture distinguishes between cues that are necessary or unnecessary for the object's presence. Computationally expensive and inexpensive modalities are also handled differently to minimise cost. The method provides a formal, tractable and robust probabilistic method for simultaneously tracking multiple objects. While instantaneous inference is exact, approximation is required for propagation over time.
\end{abstract}

\section{Introduction}

Robust tracking of a single object under occlusion from visual data is difficult due to ambiguity and noise in the sensors, uncertainty in the trajectory of the object, and variations in the appearance of the object over time. The problem of noisy sensors, or sensors that generate ambiguous output from distracting objects, can be addressed through a process of Bayesian modality fusion (BMF) $[10,11]$. BMF uses a Bayesian network to probabilistically combine the outputs of several complementary modalities. A reliability indicator for each modality is incorporated in the network. The use of complementary modalities overcomes the problem of ambiguity if a distracting object in one modality is not present in another. The problems of noisy or failing sensors are addressed through the use of probabilities and reliabilities. Uncertainty in object trajectory is generally an unsolvable problem since often we cannot know the intentions of the object (if it is a person, for example). All that can be done is to impose a general temporal model, and use global searching of the spatial domain for focusing cues rather than local searching. Unfortunately local searching is often used because global searching is necessarily computationally prohibitive. Finally the varying appearance of the object must be approached by making the chosen modalities invariant to these appearances. For example, motion and colour are generally consistent over varying appearance.

One difficulty with modality fusion is that the existing implementation [10] uses discrete variables to model the spatial domain. Each spatial variable $X$ can take the values $1, \ldots, N$, where $N$ is the number of pixels in the image. Hence marginalisation over conditional probability distributions involving spatial variables has an undesirable $\mathcal{O}\left(N^{2}\right)$ complexity. The problem can be managed to some extent by excessively sub-sampling the image domain. The consequences are not as bad as one might first think since the probability values in the sub-sampled domain are continuous. However, Toyama and Horvitz [10] do not address the problem of choosing a sufficiently accurate level of subsampling. Furthermore, the discretisation of the spatial domain prohibits extensibility to larger spatial domains and tracking of multiple objects.

Another limitation of the BMF approach in [10] is that observations of object positions are entered as specific localised evidence from an isolated tracker, ie: the observations are uni-modal. However, this is undesirable given that the combination of uncertain object trajectory and ambiguity in the modalities can result in multiple feasible observations. Much information is discarded at an early stage that could have been valuable later. Contemporary tracking approaches such as CONDENSATION [4] suffer from the same problem in a different way: only a sub-set of the current observations are used for tracking, that sub-set being determined by a temporal model. This approach contravenes the recent wisdom that successful vision requires both data-driven and model-driven processing simultaneously. To that end, the full set of observations needs to be considered simultaneously, combined with prior information, and the most likely joint hypothesis inferred, provided it is computationally tractable.

To track multiple objects, an exclusion principle must be applied on the observations so that multiple object trackers 
do not continually claim responsibility for the same observation [6]. There is generally a combinatorial explosion in the number of matching possibilities over time. Previous approaches at explicitly tracking multiple objects $[7,3,9,5]$ have used heuristic approaches to deal with this complexity.

We propose a new Bayesian modality fusion, Continuous Global Evidence-Based Bayesian Modality Fusion (CBMF), that makes four novel contributions but is also computationally tractable: 1) Continuous sampling: the formerly suggested discrete domain spatial variables [10] are turned into continuous variables to assuage the complexity of inference. 2) Global evidence: all observations from a single modality are considered during inference rather than a single position decided upon at an early and premature stage. 3) Distinct Modality Types: a distinction is made between modalities that are necessary for the presence of an object and those that only hint at its presence. 4) Selective Computation: computationally expensive modalities are treated differently from inexpensive modalities to improve performance. The network architecture is modularly expanded to simultaneously track multiple objects and impose an exclusion principle in a theoretically principled manner that exploits Bayesian "explaining away" [8]. Although exact inference in the continuous spatial domain can be used at a given time instant, an approximation is inevitably required for propagation over time.

\section{CBMF for a Single Object}

General propagation rules in singly-connected Bayesian Networks for mixture-of-Gaussian variables have previously been derived in [2]. Here we present a formulation for the specific CBMF architecture. Rather than entering observations of a 2 -dimensional spatial variable $Z=\left[z_{1}, z_{2}\right]$ as a specific value, evidence is entered as a likelihood over values. The likelihood of the observational evidence $e_{Z}$ on the variable $Z$ is modelled as a mixture of $K$ Gaussians: ${ }^{1}$

$$
p\left(\boldsymbol{e}_{Z} \mid z\right)=\sum_{k=1}^{K} \alpha_{k} G\left(z ; \mu_{k}, \sigma_{k}\right) ; \quad \sum_{k=1}^{K} \alpha_{k}=1
$$

Clearly evidence must be discarded as irrelevant at some stage in the process to avoid high computational cost. However we allow this discarding process to be driven by the observations rather than some prior and possibly misconceived hypothesis. Gaussian mixtures are only defined for spatial regions in which the modality yields a non-zero response. Therefore in general, $K$ will vary from observation to observation.

Conditional probabilities between continuous variables $X$ and $Y$ are modelled using a continuous 2-dimensional

${ }^{1}$ In this paper, all 2D Gaussians have diagonal covariance, and the functional parameters $z, \mu$ and $\sigma$ are 2-vectors.
Gaussian distribution:

$$
p(y \mid x)=G(y ; x, \sigma)=\frac{1}{2 \pi \sigma_{1} \sigma_{2}} e^{-\frac{\left(y_{1}-x_{1}\right)^{2}}{2 \sigma_{1}^{2}}-\frac{\left(y_{2}-x_{2}\right)^{2}}{2 \sigma_{2}^{2}}}
$$

Therefore marginalisation over large tables of discretised Gaussian distributions is avoided through the simplified analytical form of Gaussian convolution.

The general architecture of the CBMF network is shown in Figure 1. The chief inferred node $X$ represents the position of the object. It is a continuous variable whose distribution is generally a mixture of Gaussians. $X$ nodes are conditionally dependent over time to allow for a temporal dynamic model. Modalities are divided into two classes: necessary and contingent. Necessary modalities, $Y_{k}$, must be present when an object is present, and these nodes form the set of child nodes of $X$. Contingent modalities are represented by $U_{k}$ and may or may not be present when $X$ is present. They form the set of parents of $X$. Each modality has a continuous spatial variable which is a mixture of Gaussians. Each modality also has an associated reliability node and sub-network that measures the reliability of the modality. Each reliability variable has a set of child indicator variables which serve as external information alluding to the current reliability of the modality. For example, for a motion-based modality, a suitable indicator may be the instantaneous motion energy in the image. If the energy were to drop to zero, this would indicate that the motion modality is unreliable. The reliability $R_{k}$ and associated indicators $I_{k, 1}, \ldots, I_{k, n_{k}}$ are all discrete variables. Virtual evidence is entered into each modality node using a dummy child node $e_{k}$. We exploit the global independence of variables by applying local propagation rules to determine the belief distribution for $X$ given the observations, $P(X \mid e)$.

In the remainder of this section, we derive a tractable solution to the proposed CBMF network for tracking. Given $M$ necessary modalities $Y_{1}, \ldots, Y_{M}$ and $N$ contingent modalities, $U_{1}, \ldots, U_{N}$ in a network ${ }^{2}$, evidence $e_{Y}=\left\{e_{Y_{1}}, e_{I_{1,1}}, \ldots, e_{I_{1, n(1)}}, \ldots\right.$, $\left.e_{Y_{K}}, e_{I_{K, 1}}, \ldots, e_{I_{K, n(K)}}\right\}$ (similarly for $e_{U}$ ) is entered via specific values $e_{I_{k, j}}$ for the indicators $\mathbf{I}$, and likelihoods $e_{Y_{k}}$ for the $Y_{k}$ (see Eqn.(1)). The posterior distribution for $X$ is:

$$
\begin{aligned}
P(X \mid e) & =P(e \mid X) P(X) / P(e) \\
& =\beta P\left(e_{X}^{-} \mid X\right) P\left(X \mid e_{X}^{+}\right)
\end{aligned}
$$

where $e_{X}^{+}$is all evidence contained in the parent sub-trees of $X, e_{X}$ is evidence contained in child sub-trees of $X$, and $\beta=P\left(e_{X}^{+}\right) / P(e)$, an irrelevant constant. We have exploited the independence of the parent and child evidences.

\footnotetext{
${ }^{2}$ Note that only one necessary modality $Y_{k}$ and one contingent modality $U_{k}$ are shown in Figure 1 for simplicity.
} 


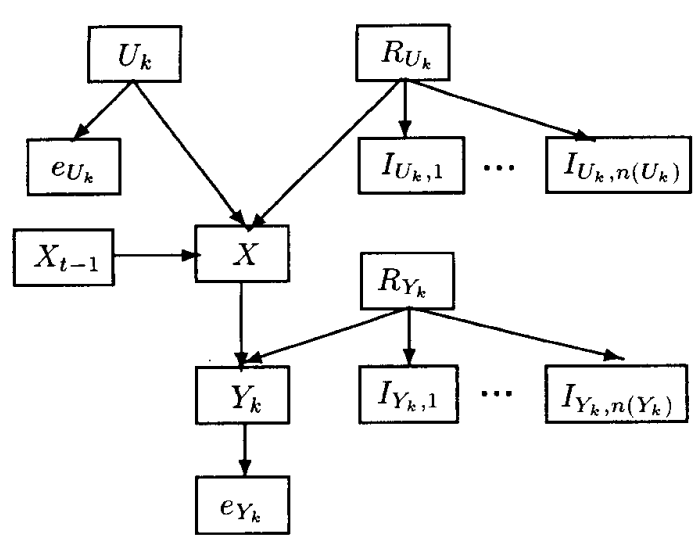

Figure 1. Continuous Global Evidence-based Modality fusion network. Only one necessary modality $Y_{k}$ and one contingent modality $U_{k}$ are shown for simplicity.

Now we can determine the two contributions separately:

$$
P\left(e_{X}^{-} \mid x\right)=\prod_{i=1}^{M} P\left(e_{X Y_{i}}^{-} \mid x\right)
$$

where $e_{X Y_{i}}$ is the evidence down the $i$ th sub-tree only. Considering $Y_{i}$ and dropping the $i$ subscript for simplicity, we can derive the contribution from each sub-tree. We define a reliability weighting function $F(\cdot)$ for convenience:

$$
F(r)=p\left(r \mid e_{R Y}^{+}\right)=\zeta p(r) \prod_{j=1}^{n} p\left(i_{j}=e_{i_{j}} \mid r\right)
$$

where $\zeta=1 / p\left(e_{R Y}^{+}\right)$. Several steps which exploit the independencies in the network yield:

$P\left(e_{X Y}^{-} \mid x\right)=\gamma \sum_{r} F(r) \int_{y} \sum_{k=1}^{K} \alpha_{k} G\left(y ; \mu_{k}, \sigma_{k}\right) G\left(y ; x, \sigma_{r}\right) d y$

where $\gamma=p\left(e_{R Y}^{+}\right)$, and $\sigma_{r}$ is the standard deviation corresponding to the given level of reliability, ie: low reliability needs high standard deviation in position for this modality. Given the 2-dimensional Gaussian functions on the domain $\left[x_{1}, x_{2}\right]$ with mean vector $\left[\mu_{i, 1}, \mu_{i, 2}\right]$ and diagonal covariance $\operatorname{diag}\left[\sigma_{i, 1}^{2}, \sigma_{i, 2}^{2}\right]$, we apply the identity: $\int_{x} G\left(x ; \mu_{1}, \sigma_{1}\right) G\left(x ; \mu_{2}, \sigma_{2}\right) d x=$ $G\left(\mu_{2}-\mu_{1} ; 0, \sqrt{\sigma_{1}^{2}+\sigma_{2}^{2}}\right)$ which yields a mixture of Gaussians:

$$
P\left(e_{X Y}^{-} \mid x\right)=\gamma \sum_{r} F(r) \sum_{k=1}^{K} \alpha_{k} G\left(x ; \mu_{k}, \sqrt{\sigma_{k}^{2}+\sigma_{r}^{2}}\right)
$$

At the $X$ node, these necessary nodes result in a product of mixtures of Gaussians. Now we address incorporation of the contingent modalities. Let us define $\mathbf{U}=\left\{U_{1}, \ldots, U_{N}\right\}$ and $\mathbf{R}=\left\{R_{1}, \ldots, R_{N}\right\}$. Then:

$$
\begin{gathered}
P\left(x \mid e_{X}^{+}\right)=\int_{\mathbf{U}, \mathbf{R}, x_{t-1}} p\left(x \mid \mathbf{u}, \mathbf{r}, x_{t-1}\right) p\left(x_{t-1} \mid e_{x_{t-1}}^{+}\right) \\
\prod_{i=1}^{N} p\left(u_{i} \mid e_{X U_{i}}^{+}\right) p\left(r_{i} \mid e_{X R_{i}}^{+}\right) d \mathbf{u} d \mathbf{r} d x_{t-1}
\end{gathered}
$$

Since the modalities are not necessarily present at the object location, we need a noisy-OR type rule. Given the modality locations, an object could really be anywhere, but is more likely to be found where the modalities occur. As modalities are superimposed, the likelihood of finding the object at a given location should increase since we have more evidence to that effect. These considerations can be represented as:

$$
p\left(x \mid \mathbf{u}, \mathbf{r}_{;} x_{t-1}\right)=\delta+w p\left(x \mid x_{t-1}\right)+w \sum_{i=1}^{N} p\left(x \mid u_{i}, r_{i}\right)
$$

where $\delta$ is a constant signifying that the object could be anywhere, $w=(1-\delta) /(N+1)$ is a weighting giving equal favour to all modalities, and $p\left(x \mid x_{t-1}\right)$ represents the temporal model for evolution of object position. Note that the distribution's expectation over $X$ must equal one, hence the weightings $w . \delta$ represents the extent to which no contingent modality can give indication as to the object's whereabouts. For example, for a motion-based modality, $\delta$ would represent the proportion of image frames in which no motion occurs. For a face detection modality, $\delta$ would represent the on-line failure rate of the face detector in a typical sequence. Such a parameter can be estimated off-line from data. Substituting Eqn. (8) into Eqn. (7), assuming uniform priors on $U_{i}$, defining $\Delta x_{t-1}$ and $\sigma_{t-1}$ to be the position offset and dispersion specified by the temporal model, and considering the prior distribution of $X$ to be a mixture of Gaussians:

$$
p\left(x_{t-1} \mid e_{x_{t-1}}^{+}\right)=\sum_{k=1}^{T} \alpha_{t-1, k} G\left(x_{t-1} ; \mu_{t-1, k}, \sigma_{t-1, k}\right)
$$

we obtain:

$$
\begin{aligned}
& P\left(x \mid e_{X}^{+}\right)=\delta+ \\
& w \sum_{k=1}^{T} \alpha_{t-1, k} G\left(x ; \mu_{t-1, k}+\Delta x_{t-1}: \sqrt{\sigma_{t-1}^{2}+\sigma_{t-1, k}^{2}}\right) \\
& +w \sum_{r_{i}} F\left(r_{i}\right) \sum_{i=1}^{N} \sum_{k=1}^{K_{i}} \alpha_{i, k} G\left(x ; \mu_{i, k}: \sqrt{\sigma_{r_{i}}^{2}+\sigma_{i, k}^{2}}\right)
\end{aligned}
$$

The final result is obtained by substituting Eqns. (4) and (9) into (3), and is a product of mixtures of Gaussians. 
The consequent exponential growth in the number of Gaussian terms is characteristic of a method that evaluates multiple joint hypotheses.

\section{Querying Expensive Modalities}

Modalities such as frame differencing and skin colour classification are inexpensive to compute and can be acquired for each pixel in the image. Other modalities, such as face detection and ellipse fitting, are not only expensive to compute, but rely on a size parameter that adds a search to the computation. It would be computationally infeasible to compute these expensive cues at each pixel for real-time applications. The modality fusion approach here can be used to selectively calculate the more expensive modalities. It is a property of Bayesian networks that evidence need only be entered in a sub-set of variable nodes at any given time. We can begin by entering evidence $e_{C}$ for the inexpensive modalities, resulting in a posterior $P\left(x \mid e_{C}\right)$. Now if there is a clear maximum in the distribution, no further computation is required. However if there is some ambiguity as to the maximum of $P\left(x \mid e_{C}\right)$, evidence $e_{F}$ can be gathered from the expensive modalities at the candidate locations. Propagation of this evidence should disambiguate the result. The criterion used in this work to query expensive modalities is to calculate at the set of local optima on $P\left(x \mid e_{C}\right)$. The new expensive evidence $e_{F}$ is then propagated to yield $P\left(x \mid e_{T}\right)=P\left(x \mid e_{C}, e_{F}\right)$, where $e_{T}$ is the total evidence.

\section{CBMF for Multiple Objects}

Now consider the case in which there are $L$ objects to be tracked in the scene. Here we present an Extended CBMF network to track multiple objects simultaneously. When tracking the objects from visual stimulus there will generally be two types of modalities: those that indicate the presence of all objects (eg: motion), and those that identify a single object (eg: appearance). The architecture is shown in Figure 2. We assume that the single object network described in section 2 has already been used to fuse modalities that are common to all objects. Therefore the posterior distribution $P\left(X \mid e_{T}\right)$ obtained from the single-object network in Figure 1 represents the probability of there being some unlabelled object at a given position. The node $X$ is then cut-and-pasted into the multi-object network of Figure 2 , such that $P\left(X \mid e_{T}\right)$, is treated as the observation for this network. Note that it is a simplifying assumption to isolate $X$ in this manner from the variables of the single-object network.

$X$ has a set of parents $\mathbf{A}=\left\{A_{1}, \ldots, A_{L}\right\}$ which are continuous variables each representing the position of an object. The figure shows the relevant variables for
$A_{i}$, the position of the $i$ th object. In similar fashion to the previous CBMF network, each $A_{i}$ has a set $\mathbf{Y}_{A_{i}}=$ $\left\{Y_{i, 1}, \ldots, Y_{i, n\left(A_{i}\right)}\right\}$ of object-specific modalities and associated reliabilities that are instantiated with mixtures of Gaussians and reliability indicator observations. The conditional probabilities associated with these object-specific modalities are the same as in the previous network. However, to facilitate explaining away by the object variables, the conditional probability table for $X$ is different, using a Noisy-OR rule [8]:

$$
p(x \mid \mathbf{A})=\eta(\mathbf{A})\left(1-\prod_{i=1}^{L}\left(1-\delta\left(x ; a_{i}\right)\right)\right)
$$

where $\eta(\mathbf{A})$ is a normalising constant ensuring that the distribution integrates to unity for a specific configuration of $\mathbf{A}$, and $\delta(x ; y)$ is the unit delta function:

$$
\delta(x ; y)= \begin{cases}1 & \text { if } x=y \\ 0 & \text { otherwise }\end{cases}
$$

For now, we ignore the normalising constant and let $\eta(\mathbf{A})=$ 1. The constant will only be different from $1 / L$ for configurations of $\mathbf{A}$ in which $a_{i}=a_{j}$ for some $i$ and $j$ in $[1, L]$. Ignoring these cases means that hypotheses regarding occluding objects are incorrectly weighted. However, ignoring $\eta$ greatly simplifies the analysis.

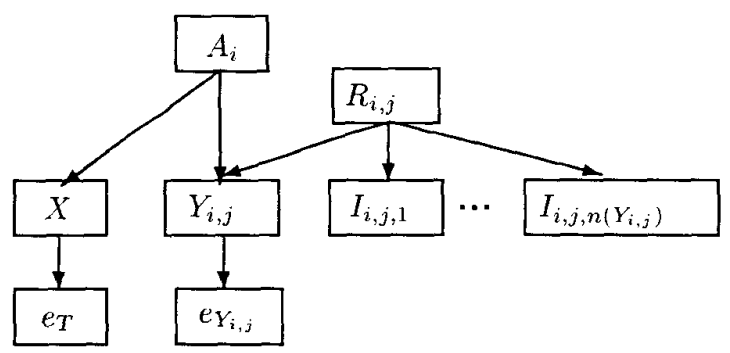

Figure 2. Unit of multi-object tracking BBN. In general there are $L$ objects, $A_{1}, \ldots, A_{L}$, and each object $i$ has $n\left(A_{i}\right)$ associated modality sub-networks. For simplicity only one object and one associated modality are shown here.

Inference using Eqn. (10) is now somewhat more complex because to infer the distribution of object $A_{i}$, information must be gathered from all $A_{j \neq i}$ through $X$. In this way objects are able to claim evidence at $X$ through the NoisyOR rule. Let the evidence entered into the multi-object network be $e_{S}$. We can determine the posterior $P\left(a_{i} \mid \epsilon_{T}, e_{S}\right)$ for each $A_{i}$ as:

$$
P\left(a_{i} \mid e_{T}, e_{S}\right)=\alpha p\left(a_{i}\right) p\left(e_{A_{i} X}^{-} \mid a_{i}\right) \prod_{j=1}^{n\left(A_{i}\right)} p\left(e_{Y_{i, j}} \mid a_{i}\right)
$$


where $\alpha=1 / p\left(e_{T}, e_{S}\right)$. The evidences $p\left(e_{Y_{i, j}} \mid a_{i}\right)$ from the object-specific modalities are computed in analogous manner to Eqn. (6). The prior $p\left(a_{i}\right)$ is important and can be taken as the posterior position distribution from the previous time frame, which may be a single Gaussian for example. The difficult term is $p\left(e_{\vec{A}_{i} X} \mid a_{i}\right)$ which relies on the other objects being tracked:

$$
\begin{aligned}
& p\left(e_{A_{i} X} \mid a_{i}\right)= \\
& \quad \beta \int_{x} \int_{A_{j}: j \neq i} p\left(e_{X}^{-} \mid x\right) p(x \mid \mathbf{a}) \prod_{j \neq i} p\left(a_{j} \mid e_{A_{j} X}^{+}\right) d A_{j} d x
\end{aligned}
$$

where $\beta=p\left(e_{A_{j}: j \neq i}^{+}\right)$is a normalising constant, $p\left(e_{X}^{-} \mid x\right)=$ $p\left(x \mid e_{T}\right)$ is the posterior from the CBMF network, treated here as evidence and generally a mixture of Gaussians, and $p\left(a_{j} \mid e_{A_{j} X}^{+}\right)$is the evidential support provided by object $j$ :

$$
p\left(a_{j} \mid e_{A_{j} X}^{+}\right)=\frac{p\left(a_{j}\right) \prod_{k=1}^{n\left(A_{j}\right)} p\left(e_{A_{j} Y_{k}} \mid a_{j}\right)}{p\left(e_{A_{j} X}^{+}\right)}
$$

Substituting Eqn. (10) into the above expression and simplifying yields:

$$
\begin{aligned}
p\left(e_{A_{i} X}^{-} \mid a_{i}\right)=\beta[1 & +p\left(e_{X}^{-} \mid x=a_{i}\right) \frac{\prod_{j=1}^{L} m_{j}\left(a_{i}\right)}{m_{i}\left(a_{i}\right)} \\
& \left.-\int_{x} p\left(e_{x}^{-} \mid x\right) \frac{\prod_{j=1}^{L} m_{j}(x)}{m_{i}(x)} d x\right]
\end{aligned}
$$

where $m_{j}(x)=1-p\left(a_{j}=x \mid e_{A_{j} X}^{+}\right)$is the object map for object $j$.

\section{The Issue of Tractability}

Although the general theory for probabilistically fusing continuous-domain quantities has been presented, there are a few issues that must be considered for a computationally tractable implementation. Each observation is represented as a mixture of Gaussians. In the simplest case, the observation could be a single Gaussian, as was the case in [10]. A more general method could use individual modality trackers to obtain an economical mixture of Gaussians using traditional techniques such as $\mathrm{K}$-means clustering or the EM algorithm [1]. In the examples presented here, observations are represented as a dense mixture of Gaussians to overcome the problems of fitting mixtures of Gaussians to data. The given modality is thresholded to remove pixels with low probability. The remaining pixels are each instantiated as the mean of a single Gaussian, with variance arbitrarily set to 1 in the $x$ - and $y$-directions, and a weighting coefficient in proportion to the modality strength. The weightings are normalised to sum to 1 . This is essentially the same as
Parzen windowing for non-parametric estimation of the observation likelihood.

The most significant remaining issue is that mixtures of Gaussians are multipled during inference, resulting in an exponential growth in their number. Consider the posterior of $X$ in the single-object tracker. The contingent modalities result in $R . N . \bar{K}$ Gaussians, where $\bar{K}$ is the average number of observation Gaussians per modality, and $R$ is the number of discrete reliability values. The necessary modalities produce the product of $M$ mixtures each containing $R \bar{K}$ Gaussians. Therefore the overall number of Gaussians is $\mathcal{O}\left(N(R \bar{K})^{M+1}\right)$. At a given time instant, the number of Gaussians can be kept to a manageable level through the observations by restricting $\bar{K}$. However, propagation over time will cause the Gaussian count to grow without bound. Therefore some approximation is required. Two possible schemes are considered. The first is to discretise the spatial posterior distribution after continuous inference, then reform an analytic distribution for propagation. One method for forming the analytic distribution is to use each local optimum in the discretised distribution as the centre for a Gaussian. The second approximation method is to carefully manage the number of analytic Gaussians by culling those that have small weighting coefficients. Here we use the discretisation approximation since it simplifies manipulation and interpretation of the distribution. For example, the most common use of $P(X \mid e)$ is to find the value of $X$ that maximises the distribution: $x^{*}={ }_{x}^{\operatorname{argmax}} P(x \mid e)$. However, there is no straight-forward way to maximise a superposition of Gaussians ${ }^{3}$. Given the discretised distribution, $x^{*}$ can be easily determined.

It is worth noting the comparison in computational expense between our approach using discretisation and other tracking methods. Let $D$ be a measure of the extent of the spatial domain being modelled. For instance, $D=w \times h$ would be the number of pixels in an image. In the case of tracking a single object, the complexity of CBMF is $\mathcal{O}(M \bar{K} D R)$, where $M$ is the total number of modalities. Therefore computation is linear in the number of modalities, observation units and domain size. Compare this with the original BMF framework which used discrete spatial variables. In this case, $\bar{K}=1$ because only one observation hypothesis was used per modality. The complexity of inference is $\mathcal{O}\left(M \bar{K} D^{2} R\right)$, which is quadratic in domain size! The saving has come about through exploitation of the simple analytic form of the convolution of two Gaussians. The other improvement is that our method allows $\bar{K}>1$ so that valuable low-level information is not discarded during high-level inference.

\footnotetext{
${ }^{3}$ In general the local maxima of the superposition will occur when the sums of derivatives of terms equals zero:

$$
\frac{d F(x)}{d x}=0=\sum_{i} \alpha_{i} \frac{d G\left(x_{;} \mu_{i}, \sigma_{i}\right)}{d x}
$$
}


For the case of tracking $L$ objects, the complexity of $\mathrm{CBMF}$ is $\mathcal{O}(L \bar{M} \bar{K} D R)$ where $\bar{M}$ is the average number of object-specific modalities. Therefore the complexity is the same as for our single-object case, but scales linearly with $L$. This is a profoundly important property for simultaneous tracking of multiple objects: the usual combinatorial explosion in joint object location hypotheses is avoided by communication through the $X$ node. By comparison, other approaches such as [5] retain the $L^{2}$ complexity and assume tractability due to a small number of objects. Our approach can be compared with partitioned sampling [6], in which a hierarchical model of object independence is exploited to avoid $L^{2}$ complexity. However, our approach is deterministic, does not suffer from sparse sampling problems and has fixed computational complexity.

For multi-object tracking, note that the last term in Eqn.(12) is a constant over $a_{i}$. Therefore a discretised object map can be calculated for each object. A combined map $\prod_{j=1}^{L} m_{j}(x)$ for $X$ can then be computed. For a specific object, the maps are combined with the observation at $X$ to determine $p\left(e_{A_{i} X}^{-} \mid a_{i}\right)$.

\section{Experimental Results}

We tested the CBMF approach on the problem of tracking an individual's head in a video sequence. Three modalities were used: skin colour (necessary), frame differencing (contingent), and ellipse fitting (necessary). While skin and motion are cheap to compute, the fitting of an ellipse to an edge image is expensive since the head position and size must be first hypothesised. Therefore the network used had $\mathbf{Y}=\left\{Y_{1}, Y_{2}\right\}$ where $Y_{1}$ is skin colour and $Y_{2}$ is the ellipse fit, and $\mathbf{U}=\left\{U_{1}\right\}$ where $U_{1}$ is the motion estimate. The ellipse fitting modality was queried as an expensive modality. We used a broad Gaussian distribution for the temporal model to specify the object's expected position at the next time step, with $\Delta x_{t-1}=0$. Three discrete reliability values, low, medium and high, are used in the network. Similarly all reliability indicators are discretised to one of the three values low, medium and high.

The cues were calculated as follows. The frame difference is the absolute difference between consecutive greyscale images. The skin image was computed using a single multi-dimensional Gaussian for classification in normalised RG-colourspace, where $R=r / N, G=g / N$, and $N=r+g+b$. The Gaussian parameters were estimated off-line using user-selected image regions. In the cases of motion and skin colour, these real-valued images were then thresholded to obtain a binary classification. At the queried image locations, the ellipse fit was obtained on a blurred edge image at multiple sizes on the range of 20 to 60 pixels in width. An ellipse aspect ratio of $x: y=1: 1.2$ was assumed. The criterion used is $f=s / n$, where $s$ is the number of non-zero edge pixels under the ellipse perimeter, and $n$ is the total number of perimeter pixels. The ellipse size with the highest criterion value at that position was used.

The reliability indicator for the motion cue was the number of moving pixels in the image, the rationale being that when there is either virtually zero or a great deal of motion present, that cue is unreliable for identifying the head. For skin, two indicators were used. The number of skin pixels was used in similar manner to the number of motion pixels. The second indicator is here termed pearling, or patchiness of the skin image. It is computed as the average variance of the binary skin image in $3 \times 3$ tiles. The more patchy the skin image is, the less reliable this modality. No reliability indicator was used for the ellipse fitting modality. $\delta$ in Eqn. (8) was arbitrarily set to 0.1 .

A sample frame from results on a test sequence is shown in Figure 3. The figure shows (from left to right, top to bottom) the original image, the motion image, the skin image, the motion modality observation as a mixture of Gaussians, the skin modality observation, the prior distribution of $X$, the intermediate posterior $P\left(X \mid e_{C}\right)$, the expensive ellipse fitting modality observation instantiated at the appropriate locations, and the final fused distribution $P(X \mid e)$. It can be seen that many hypotheses for the position of the head are considered by the tracker. In the initial fusion result there are two competing peaks, one corresponding to the hand and the other to the head. The expensive ellipse fit modality is queried at the local maxima of the fused distribution and propagated to yield the final fused posterior. The expensive modality has successfully disambiguated the head with a clear peak at the proper location.

In the second example, $\mathrm{CBMF}$ is used to simultaneously track the heads of three people under occlusion. The same experimental configuration as the first example was used to obtain a distribution on $X$, based on skin colour classification, frame differencing and ellipse fitting. The skin colour model used was trained using pixels from all three individuals. The single person-specific modality for each object was a skin classification based on a person-specific colour model. Reliabilities were used for the object-specific colour models as in the previous experiment. A sample frame is shown in Figure 4, and the CBMF results are shown in Figure 5. The figure shows the person-specific skin colour modalities as mixtures of Gaussians on the first row, and the final posterior distribution for each object position on the second row. The results are startling. The modes in the posterior distributions match the positions of the correct faces for the respective skin colour model. However examining the figure it can be seen that only the modality distribution for the first object is very distinctive for that object. Nevertheless, the mechanism of Bayesian "explaining away" has ensured that the second and third objects cannot be found at the distinctive position of the first object. 

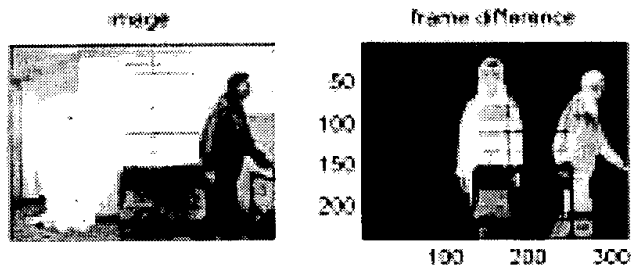

mogaly cto. Twith - cortinged
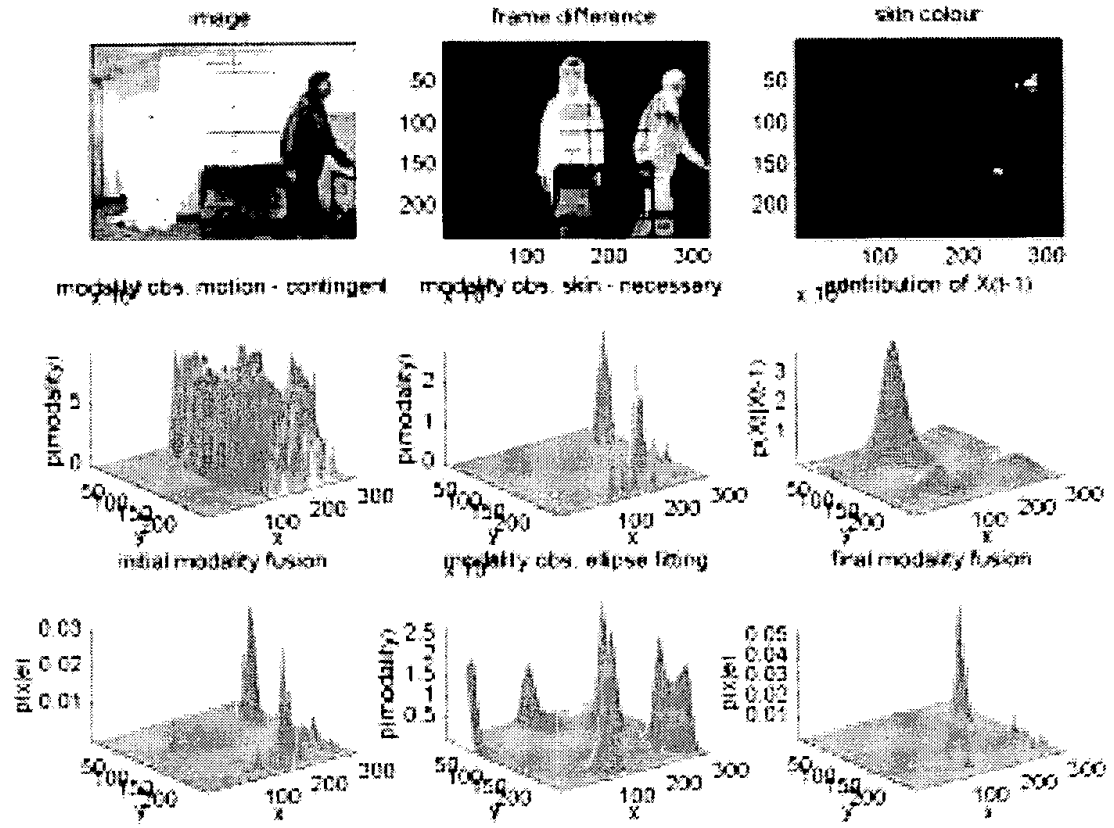

Figure 3. Continuous Global Evidence-Based Bayesian Modality Fusion results from a sample frame in a sequence. The figure shows from left to right, top to bottom: the original image, the motion image, the skin image, the motion modality observation as a mixture of Gaussians, the skin modality observation, the prior distribution of $X$, the intermediate posterior $P\left(X \mid e_{C}\right)$, the expensive ellipse fitting modality observation, and the final fused distribution $P\left(X \mid e_{T}\right)$.

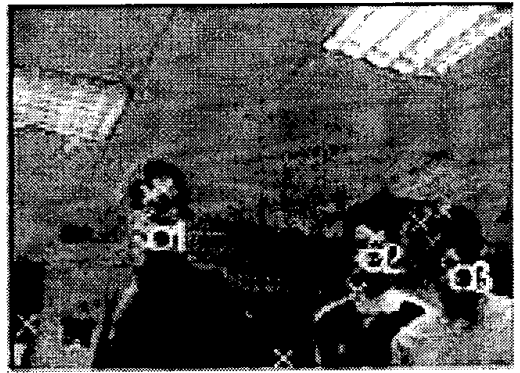

Figure 4. Sample frame from multi-object tracking example. Crosses show positions of local optima in $P\left(X \mid e_{T}\right)$, and labelled circles show estimated object positions.

\section{Conclusion}

We have presented a theoretically sound, computationally tractable, comprehensive probabilistic framework for continuous-valued, global evidence-dependent Bayesian modality fusion to track multiple objects in space. For tracking multiple objects simultaneously, the model complexity grows linearly with the number of objects rather than quadratically as for some existing techniques. The method uses exact inference, is deterministic, and combines information globally from all observations with prior information propagated over time. The method can distinguish between necessary and contingent modalities, and between computationally expensive and cheap visual cues.

This method is a recent development and can be improved in a number of ways. A more efficient implementation may be developed that is able to quickly find the local maxima of a mixture of Gaussians. This would remove the reliance of the computational complexity on the spatial 

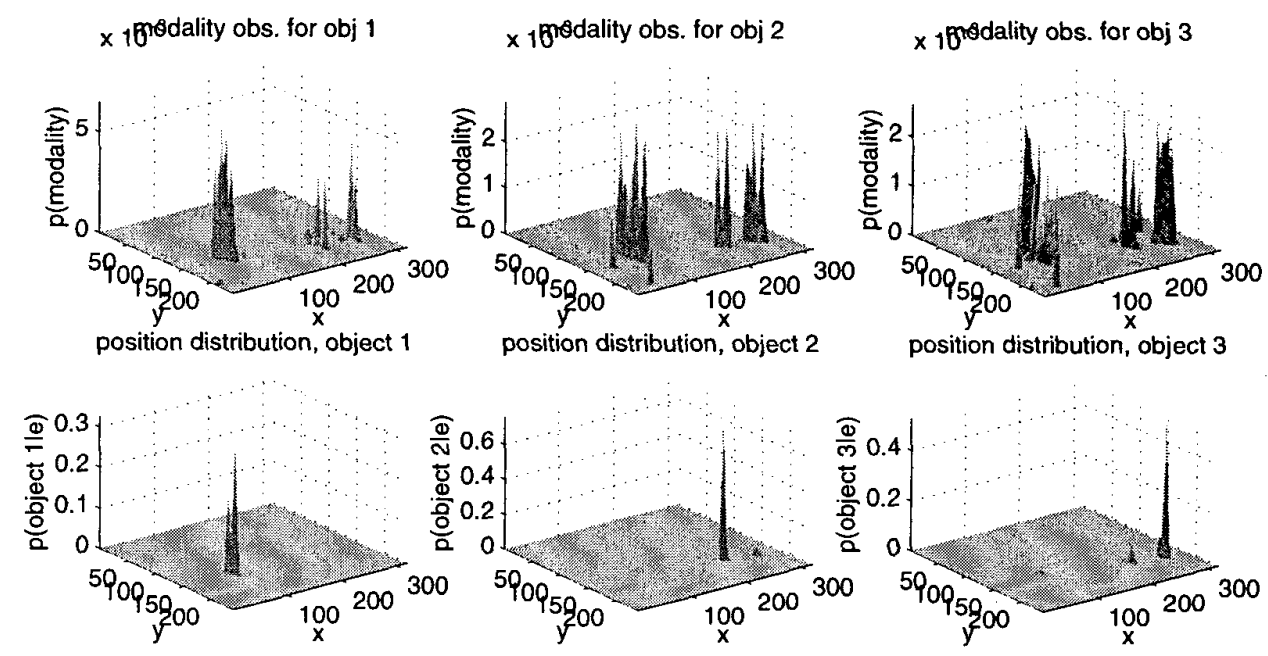

Figure 5. CBMF tracking three heads simultaneously; results on a sample frame from a sequence. The top row shows observations as mixtures of Gaussians. The bottom row shows the posterior distribution of position for each object. The corresponding image frame is shown in Figure 4.

domain size. Certain parameters in experiments have been selected in an ad hoc manner, but could be estimated from data. Currently the CBMF network does not explicitly handle the case that the object leaves the field of view. The architecture will need to be modified to handle this case.

The temporal dependence between object location distributions in the multi-object tracker has been simplified by copying the posterior at time $t$ to be the prior at time $t+1$ because inclusion of these connections would overcomplicate inference. It remains to be seen whether proper inclusion of these dependencies can improve tracking. Another issue with the multi-object tracker is that the number of objects being tracked is currently fixed over time. We are experimenting with the following solution to this problem. Tracking begins with a single object. Using Bayesian model selection techniques, the addition or removal of an object can be hypothesised periodically. Three networks are periodically tested: one with $L$ objects, one with $L-1$ and one with $L+1$. A difficult issue occurs with removal of an object, since knowing which object to remove may require consideration of $L$ new networks.

\section{References}

[1] C. Bishop. Neural Networks for Pattern Recognition. Cambridge University Press, 1995.

[2] E. Driver and D. Morrell. Implementation of continuous Bayesian networks using sums of weighted Gaussians. In Proc. Uncertainty in AI, pages 134-140, August 1995.
[3] I. Haritaoglu, D. Harwood, and L. Davis. $W^{4}$ : Real-time surveillance of people and their activities. IEEE PAMI, 22(8):809-830, August 2000.

[4] M. Isard and A. Blake. CONDENSATION-conditional density propagation for visual tracking. IJCV, 29(1):5-28, 1998

[5] L. Lee, R. Romano, and G. Stein. Monitoring activities from multiple video streams: Establishing a common coordinate frame. IEEE PAMI, 22(8):758-767, August 2000.

[6] J. MacCormick and A. Blake. A probabilistic exclusion principle for tracking multiple objects. In $I C C V$, volume 1 , pages 572-578, Corfu, Greece, September 1999.

[7] S. McKenna, S. Jabri, Z. Duric, and H. Wechsler. Tracking interacting people. In IEEE FG, pages 348-353, Grenoble, France, 2000. IEEE Computer Society.

[8] J. Pearl. Probabilistic Reasoning in Intelligent Systems: Networks of Plausible Inference. Morgan Kaufmann, 1988.

[9] C. Stauffer and W. Grimson. Learning patterns of activity using real-time tracking. IEEE PAMI, 22(8):747-758, August 2000.

[10] K. Toyama and E. Horvitz. Bayesian modality fusion: Probabilistic integration of multiple vision algorithms for head tracking. In Proceedings of the Fourth Asian Conference on Computer Vision, Tapei, Taiwan, January 2000.

[11] J. Triesch and C. von der Malsburg. Self-organized integration of adaptive visual cues for face tracking. In IEEE FG, pages 102-107, Grenoble, France, March 2000. IEEE Press. 